\title{
cgbind: A Python Module and Web App for Automated Metallocage Construction and Host- Guest Characterization
}

\author{
Tom A. Young, Razvan Gheorghe and Fernanda Duarte*
}

Chemistry Research Laboratory, University of Oxford, Mansfield Road, Oxford OX1 3TA, U.K.

KEYWORDS: Supramolecular metallocages, Host-Guest Complex, Computational Modelling

\begin{abstract}
Metallocages offer a diverse and underexplored region of chemical space to search for novel catalysts and substrate hosts. However, the ability to tailor such structures towards applications in binding and catalysis is a challenging task. Here, we present an open-source computational toolkit, cgbind, that facilitates the construction, characterization and prediction of functional metallocages. It employs known structural scaffolds as starting points and computationally efficient approaches for property evaluation. We demonstrate the ability of cgbind to construct libraries of cages with varied topologies and linker functionalities, generate accurate geometries (RMSD $<1.5 \AA$ to crystal structures) and predict substrate binding with accuracy on-par with semiempirical QM, all in seconds. The cgbind code presented here is freely available at github.com/duartegroup/cgbind and also via a web-based graphical user interface at cgbind.chem.ox.ac.uk. The protocol described here paves the way for high-throughput virtual screening of potential supramolecular structures, accelerating the search for new hosts and catalysts.
\end{abstract}

*corresponding author: fernanda.duartegonzalez@chem.ox.ac.uk 


\section{Introduction}

Metal-mediated self-assembly has emerged as a promising approach to develop complex biomimetic systems from simple building blocks. The synthetic modularity and tunability, achievable by modifying the metal center or organic linkers, has led to the discovery of an immense diversity of supramolecular metallocages with applications in molecular separation ${ }^{1,2}$ medical imaging, ${ }^{3,4}$ drug-delivery ${ }^{5-10}$ and catalysis. ${ }^{11}$ Moreover, the dynamic nature of metal-ligand bonds, intermediate in strength between covalent bonds and weak non-covalent interactions (NCI), facilitates error correction and reversibility. ${ }^{12,13}$ The latter being more challenging to achieve with covalent organic cages. ${ }^{14}$

Despite these attractive attributes, the development of new metallocages and their use in real-life applications has been slow, and in many cases driven by trial-and-error rather than rational design. To date, most reported binding studies have been limited to a relatively small set of guests and host combinations. This is primarily due to current synthetic techniques being time-consuming and expensive; while also limited in environmental sustainability. ${ }^{15}$ To fully exploit their potential, the development of efficient methods to identify structures with desirable binding or catalytic properties, without having to synthesize all possible structures, is necessary.

Computational screening offers a path to overcome these limitations and accelerate the discovery process. ${ }^{16}$ While reaching chemical accuracy $\left(<1 \mathrm{kcal} \mathrm{mol}^{-1}\right.$ error), generally required for quantitative comparison to experiments remains a challenge, ${ }^{17}$ current computational and cheminformatic approaches already allow a fast sift of the desired space. For example, molecular descriptors ${ }^{18-20}$ and inverse design methods ${ }^{21}$ can be used to select (with reasonable accuracy) promising regions of the chemical space for further computational and eventually experimental study. ${ }^{22}$ Similarly, efficient electronic structure methods, in particular density functional theory (DFT), have enabled the calculation of structure and properties for hundreds of compounds in a single study. ${ }^{23,24}$ While these protocols are accessible for small organic molecules and periodic structures, extension of similar approaches in supramolecular assemblies has been much less explored. ${ }^{25}$ We have recently shown that an efficient DFT-based approach can reach a $\sim 2 \mathrm{kcal} \mathrm{mol}^{-1}$ level of accuracy in quantifying binding affinities for $\mathrm{M}_{2} \mathrm{~L}_{4}$ metallocage assemblies. ${ }^{26}$ However, with a sufficiently fast computational approach, the required time to set up and analyze a new set of metallocages rapidly increases as the size of the building block library grows. Therefore, methods to automate these steps are becoming increasingly valuable.

In the field of metal-organic frameworks (MOFs), several computational screening tools have been developed in recent years. ${ }^{27,28}$ They include open-source tools to screen existing MOF libraries $\left(\right.$ Pymatgen $^{29}$ and Zeo $++{ }^{30}$ ), deconstruct known structures in their building blocks, ${ }^{31}$ and evaluate their catalytic proficiency for specific reaction (PyMOFScreen ${ }^{32}$ ). In the space of supramolecular metallocages, however, no large already synthesized libraries exist; thus, tools that both generate and rank structures for desired properties are required. For porous organic cages, in silico toolkits have been developed for constructing and predicting their adsorption properties 
via a combination of building blocks and high-throughput screening (e.g. stk, pywindow, Eigencages). ${ }^{20,33-37}$ Current toolkits to generate structures are, however, not directly transferable to metal-containing systems. Therefore, a different methodology is required to further enable high-throughput analysis of supramolecular metallocages.

Here, we describe cgbind, an open-source Python API and its web-based graphical user interface (GUI). We first describe the methodology and its implementation and proceed to demonstrate the ability of cgbind to explore the space of metallocages by combinatorial cage generation from a building block library. Furthermore, we show how cgbind can be used to generate accurate geometries and obtain estimated binding affinities. 


\section{Results and Discussion}

\subsection{Software Outline}

cgbind is written in object-oriented Python and employs scientific programming libraries, including Numpy, Scipy and NetworkX..$^{38-40}$ The code is built around four key objects (Linker, Cage, Substrate and CageSubstrateComplex), the relation and inheritance between which, their attributes, and key methods are outlined in Figure 1.

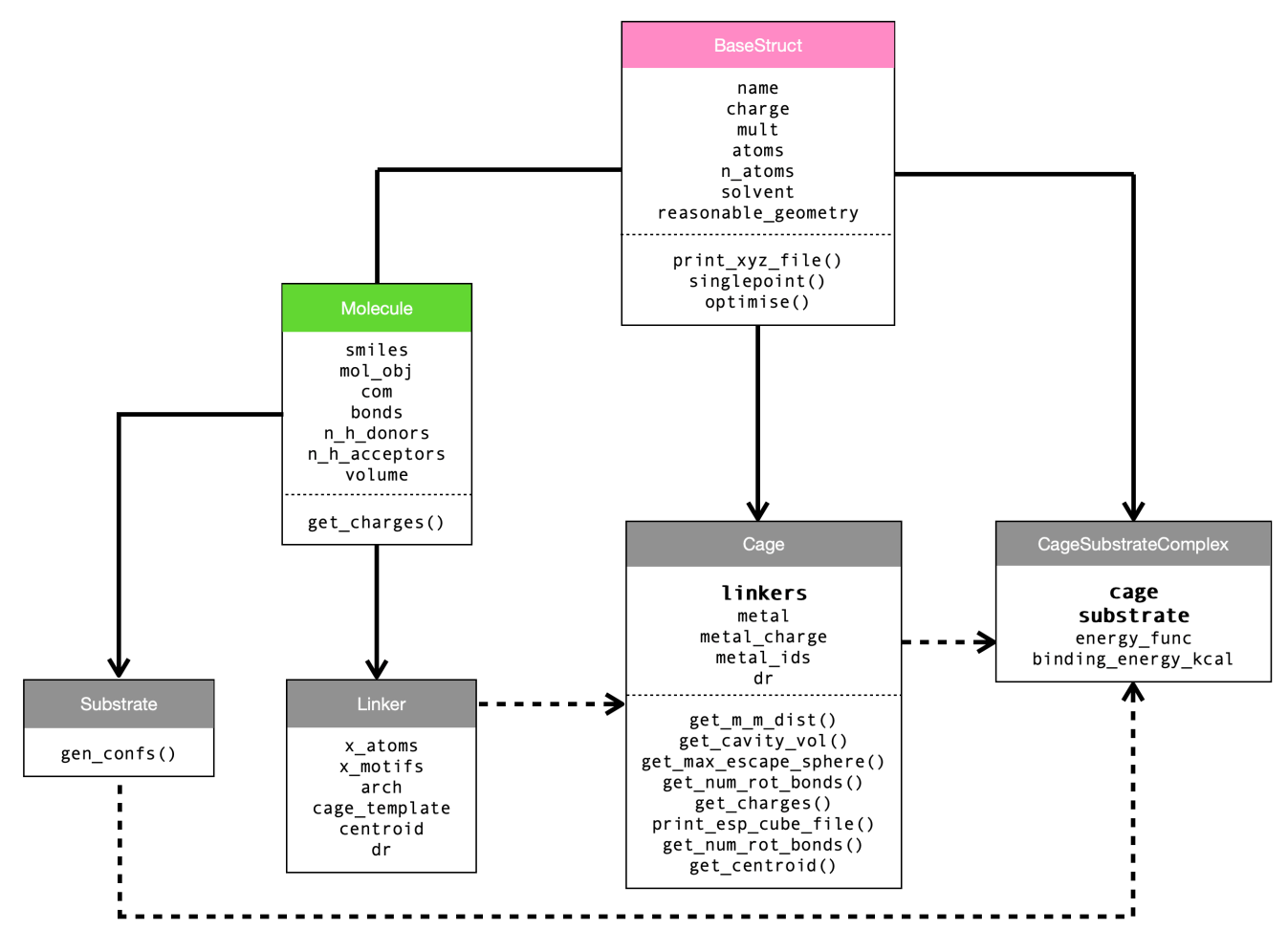

Figure 1. Class structure of cgbind. A solid line from A to B indicates that B inherits from A and a dashed line B is built from $\mathrm{A}$.

A homoleptic Cage $\left(\mathrm{M}_{x} \mathrm{~L}_{y}\right.$ with $x$ metals and $y$ linkers) is initialized from a single Linker object, while heteroleptic (e.g. $\mathrm{M}_{2} \mathrm{~L}_{2} \mathrm{~L}_{2}$ ) variants from a set of different Linkers. All Molecule objects and their subclasses are designed to be initialized from SMILES strings, ${ }^{41}$ with their corresponding 3D structures generated using conformer generation algorithms implemented in RDKit. ${ }^{42,43}$ While this affords rapid access to 3D structures, conformers are occasionally not adequately generated (vide infra). Molecules can also be initialized from a variety of molecular file formats (.xyz, .mol, .mol2) using the input_output module. Upon Cage construction a number of analysis tools are available as class methods. The analysis of cage-substrate complexes (CageSubstrateComplex objects) is also possible. They are built from a Cage and one Substrate, where a 
substrate may contain more than one molecule, thus multiple guests are supported. Furthermore, a Substrate can either be a minimum or a transition state (/analogue), providing a route to predicting catalytic activity.

Binding affinities between a substrate (S) and cage (C) $\left[\Delta E_{\mathrm{BA}}=E_{\mathrm{C}-\mathrm{S}}-\left(E_{\mathrm{C}}+E_{\mathrm{S}}\right)\right]$ are calculated in the first instance using a simple and general forcefields developed here (vide infra). More accurate estimates can be obtained from electronic structure codes, by invoking singlepoint() and optimise() functions, which deliver energies (Molecule.energy) and optimized structures (Molecule.atoms). These functions are currently handled through a Calculation object in our Python API autodE, which facilitates the automated generation of reaction profiles (github.com/duartegroup/autodE). This enables cgbind to utilize electronic structure packages with currently implemented wrappers (inc. GFN2-XTB, ${ }^{44} \mathrm{MOPAC},{ }^{45} \mathrm{ORCA}^{46}$ and $\mathrm{NWChem}^{47}$ ).

The structure of cgbind benefits from modularity, allowing analysis functions to be implemented by simply adding methods to the Cage or CageSubstrateComplex objects. The fragment approach to generating a CageSubstrateComplex allows for all the properties of the cage, and constituent linkers to be contained within a single object. Linker, Cage and Substrate objects may therefore be reloaded from a saved (e.g. Pickled) CageSubstrateComplex object, allowing for data mining within a saved library. Independent calculations are parallelized up to the user-defined number of cores set in the Config object. The software is distributed under the MIT license and is provided with unit tests and comprehensive online documentation including examples.

\subsection{Overview of cgbind}

\subsubsection{Metallocage Construction}

Broadly, metallocage structures are generated from a SMILES or 3D representation of the linkers, which are templated onto known or user defined metallocage templates. The methodology employed is not template specific, such that extension to architectures other than those provided with the code $\left(\mathrm{M}_{2} \mathrm{~L}_{4}, \mathrm{M}_{4} \mathrm{~L}_{6}, \mathrm{M}_{6} \mathrm{~L}_{8}, \mathrm{M}_{12} \mathrm{~L}_{24}\right)$ is straightforward. Figure 2 provides an overview of the workflow behind cgbind, which can be broken down into six key steps:

Step I. Generate $n$ linker (L) conformers from a SMILES string, a common way of representing molecules, which can be obtained from online databases (e.g. PubChem) or chemical software (e.g. ChemDraw $\left.{ }^{\circledR}\right)$.

Step II. The donor atoms bonded to the metal atoms ( $X$-atoms) are located in L.

Step III. Locate possible $X$-motifs, as all combinations of one or more $X$-atoms and their nearest neighbors in $\mathrm{L}$.

Step IV. Discard any $X$-motifs with a different number of atoms to the template $X$-motifs. 
Step V. Minimize the fitting cost with respect to linker \& template parameters. The fitting cost is defined as the sum squared distance (SSD) between the X-motif atoms in the template and L.

Step VI. Fit linkers to the expanded/contracted template, controlled by a distance $\Delta r$, while keeping steric clashes below a threshold.

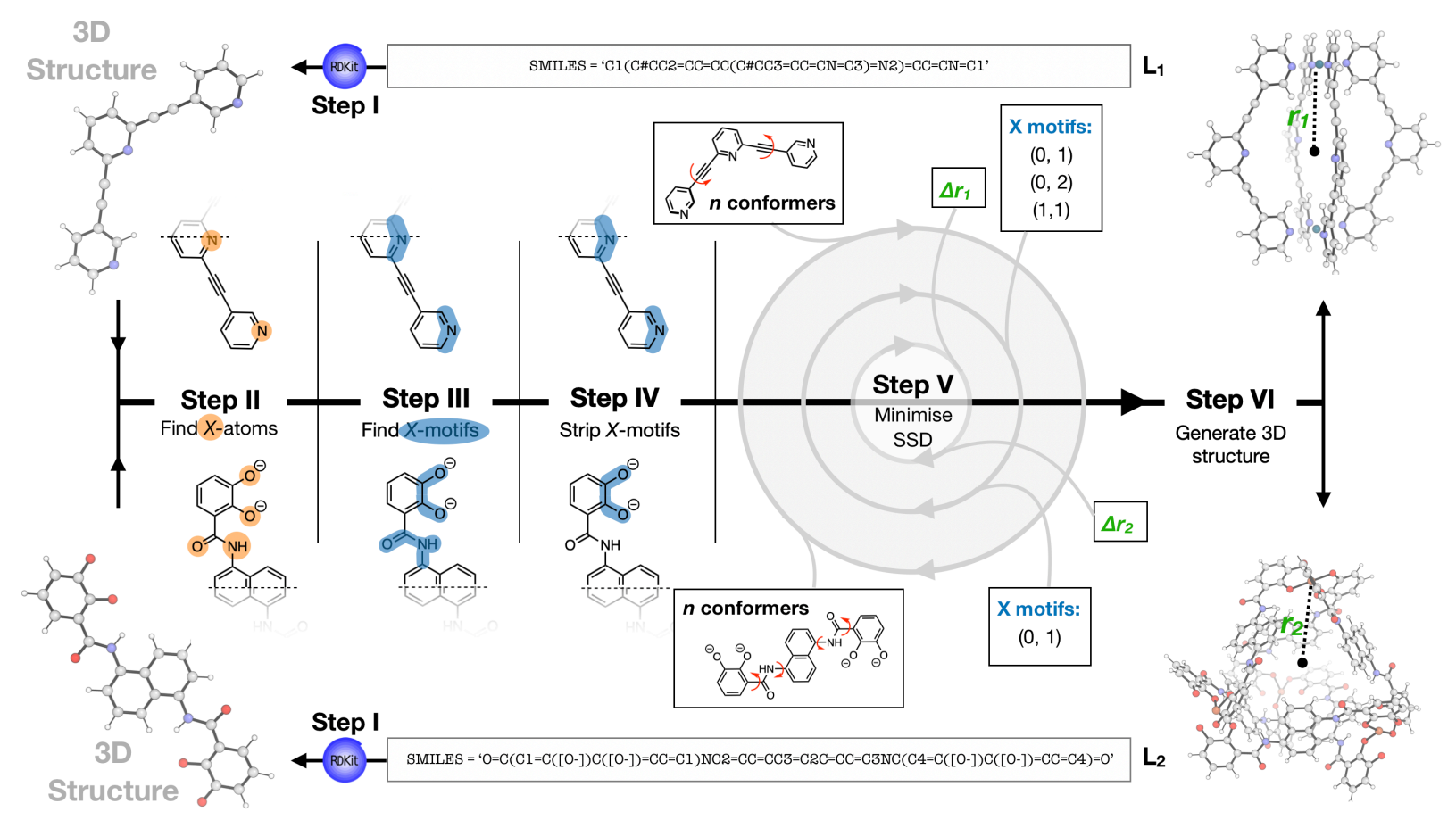

$\overline{\text { Figure 2. Methodology used to construct metallocages in cgbind. Centroid-metal distances are denoted as } r \text { and }}$ shifts $\Delta r . X$-motifs are indexed from zero to the total number in the linker.

These steps are discussed in detail below for two architectures, $\mathrm{M}_{2} \mathrm{~L}_{4}$ and $\mathrm{M}_{4} \mathrm{~L}_{6}$, already saved in the template library. Note that, depending on the input provided by the user, not all steps must be executed. For example, if a Linker is initialized from a 3D geometry then the Step I can be skipped. Moreover, if the $X$-motifs are specified by the user then steps III and IV may be skipped, as well as minimization with respect to $X$-motif sets in Step V.

For a bis-pyridyl linker found experimentally to form a $\mathrm{M}_{2} \mathrm{~L}_{4}$ assembly ( $\mathbf{L}_{\mathbf{1}}$, top Figure 2 ), ${ }^{48}$ the first step involves generation of 3D conformers using the Experimental-Torsion Distance Geometry with Knowledge (ETKDG) algorithm ${ }^{43}$ implemented in RDKit ${ }^{42}$ (Step I). For this linker, requesting 300 conformers and using a $0.3 \AA$ root mean square displacement (RMSD) threshold to compare them, renders 50 conformers. This provided a good balance between speed and high probability of locating a suitable conformer. Subsequently, $X$-atoms are identified as the heteroatoms with at least one 'lone pair' capable of metal donation (here the nitrogen atoms, Step II). Combining $X$-atoms and their nearest neighbors affords three $X$-motifs containing CNC atoms (Step III). In the $\mathrm{M}_{2} \mathrm{~L}_{4}$ template, the $X$-motifs also contain three atoms (see $\$ 2.2 .2$ Adding New Template Architectures for how these motifs are found); therefore, all $X$-motifs are kept (Step IV). Minimization of the 
fitting cost is then performed exhaustively with respect to all conformers and $X$-motif sets, and minimization with respect to expansion and contraction of the template is achieved using the Broyden-Fletcher-GoldfarbShanno (BFGS) optimization algorithm implemented in $S c i P y^{39}$ (Step V). The template is expanded/contracted by shifting the $X$-motifs closest to metal $i$ by the $\mathrm{M}_{i}-$ centroid vector a distance $\Delta r$. For $\mathbf{L}_{\mathbf{1}}$, there are three $X$ motifs (top: 0 , middle: 1 and bottom: 2 , with 0 and 2 being equivalent), while there are only two $X$-motifs in the template linker; therefore, the three $X$-motifs in $\mathbf{L}_{1}$ can be fitted in three different ways, i.e. $(0,1),(0,2)$ and $(1$, 2). $X$-motif atoms are fit to the template using an implementation of the Kabsch algorithm. ${ }^{49}$ Step V correctly identifies the linker with the most coplanar pyridyls as the optimum conformer, the two terminal CNC atom sets $(0,2)$ as the optimum selection of $X$-motifs and a small shift required in the template $\left(\Delta r_{1}=-0.0037 \AA\right)$. Upon sequentially fitting the optimum $X$-motifs in the linker to the adjusted template and adding two metal ions, a metallocage geometry is generated (Step VI).

In an $\mathrm{M}_{4} \mathrm{~L}_{6}$ structure formed from identical catechol-derived linkers and $\mathrm{Fe}^{2+}\left(\mathbf{L}_{\mathbf{2}}\right.$, Figure 2$),{ }^{50}$ steps I/II proceed as for $\mathbf{L}_{1}$, while identification of $X$-motifs is slightly more complex. $X$-atoms are joined by their nearest neighbors to generate $X$-motifs for $\mathrm{C}^{-} \mathrm{O}^{-}, \mathrm{C}=\mathrm{O}$ and $\mathrm{CN}(\mathrm{H}) \mathrm{C}$ and those separated by a one bond joined generating ${ }^{-} \mathrm{OCCO}^{-}$ and $\mathrm{O}=\mathrm{CN}(\mathrm{H}) \mathrm{C}$ motifs to give a total of 12 motifs in $\mathbf{L}_{2}$ (Step III). The template contains $X$-motifs with four atoms, thus only the ${ }^{-} \mathrm{OCCO}^{-} X$-motifs remain upon discarding those with greater and fewer than four atoms (Step IV). Steps V and VI proceed as for $\mathbf{L}_{\mathbf{1}}$ but requires no enumeration over $X$-motif sets as both $\mathbf{L}_{\mathbf{2}}$ and the template contain two $X$-motifs.

This strategy is amenable to heteroleptic cage generation; different linkers are generated within the same architecture and fitted in the order defined by the template. The template shift distance $\Delta r$ is taken as an average over all linkers, as to accommodate linkers with (slightly) differing lengths. For an experimentally accessible cis- $\mathrm{M}_{2} \mathrm{~L}_{2} \mathrm{~L}_{2}$ ' metallocage ${ }^{51}$ the process is shown in Figure 3. We note that the trans isomer may be generated by simply reordering the linkers in the Cage initialization (i.e. linkers $=[$ linker1, linker2, linker1, linker2] in Figure 3). 


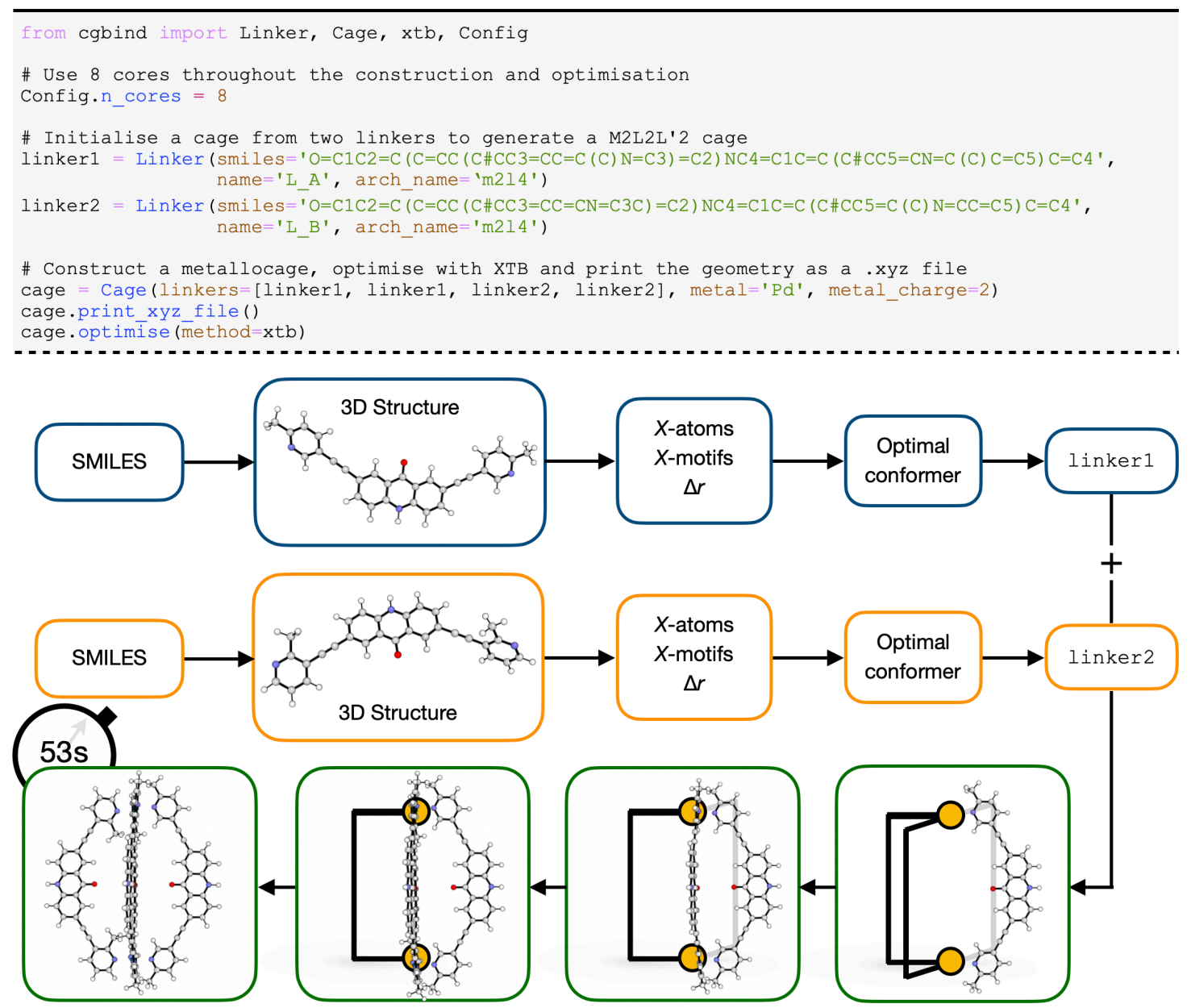

Figure 3. Formation of a heteroleptic cage within cgbind. Total execution time includes XTB optimization.

For a linker with $n$ conformers, $j X$-motifs in the template and $i$ metallocage $X$-motifs, the minimization is conducted in a $\sim n \times{ }^{i} \mathrm{C}_{j}$ dimensional space. However, despite this large space, generally it is the conformer generation in RDKit, rather than minimization which dominates the execution time. Construction of cages with non-planar and or conformationally flexible linkers is facilitated by checking on the ordering of $X$-motifs and linker conformer as to achieve a threshold in the linker-partial cage repulsion during the build algorithm.

\subsubsection{Adding New Template Architectures}

Extensibility to new architectures is an essential feature of the code. The inclusion of new architectures by the user is achieved as follows: A 3D structure file (.mol2) is used for initialization of a Template. This structure can be downloaded from the CSD, or provided by the user (from non-deposited crystal structures or e.g. Spartan ${ }^{52}$ generated constructs).

(1) Utilizing NetworkX a molecular graph of the system is generated. 
(2) A metallocage is identified as the molecular component with the largest number of metal atoms. All other molecules (ions, substrates) are stripped out; this step is required if the template is initialized from a crystal structure.

(3) The donor $(X-)$ atoms bonded to the metal atoms are located.

(4) Linkers are identified by deleting metal atoms and determining its connected components using NetworkX.

(5) Within the linkers, and from the previously located $X$-atoms, $X$-motifs are located as the maximally connected set of $X$-atoms and their nearest neighbors.

(6) The shift vector for each $X$-motif is calculated to enable symmetric expansion of the template controlled by $\Delta r$ as above.

Overall, this workflow facilitates the addition of novel architectures into the code with minimal human intervention (i.e. a single command) and without structures needed to be hardcoded.

\subsubsection{Analysis Tools}

Upon generation of a metallocage structure, cgbind provides several geometry-based parameters that can be used to analyze and select metallocages for further binding and/or catalysis studies (Figure 4a). These include:

a. Number of rotatable bonds, which may be used as a proxy for cage flexibility (by default, the M-L bonds are not included).

b. Total number of H-bond, which can be used as a proxy for hydrophilicity. They include H-bond donors (non-coordinatively saturated heteroatoms) and acceptors (hydrogens bonded to a heteroatom).

c. $\quad$ Cage size metrics. They include:

(i) Average $\mathrm{M}-\mathrm{M}$ distance.

(ii) Maximum enclosed sphere: defined as the largest sphere centered on the cage centroid that does not contain any atomic (van der Waals) volume. The cage centroid is defined as the mean position of the metal atom centers.

(iii) Maximum escape sphere: defined the largest sphere that may be removed from the cavity. This volume is determined by maximizing the sphere size at a distance $r$ from the cage centroid and taking the minimum sphere size along the path to the outside of the cage.

The latter process can be performed with a basin-hopping algorithm from the SciPy library, which provides a more accurate estimate of the extrema but is computationally demanding. ${ }^{53}$ If the cage is symmetric, initializing with a random vector generally affords the largest escape sphere. However, for asymmetric cages, the use of a basin-hopping algorithm is necessary. This methodology is similar to that employed in pywindow $w^{37}$ and may be used as a proxy for window size, and consequently, substrate diffusion rates into/from the interior of the cage. 
d. Electrostatic potentials (ESP), which can be used to identify favorable guest-host complexes by maximizing electrostatic interactions between them. By interfacing cgbind with the open-source tight binding DFT code XTB, ${ }^{44,54}$ ESPs can be obtained in seconds in the standard Gaussian cube format and visualized in PyMOL or other graphical software (Figure 4b). This is achieved by performing a single point energy evaluation on the structure, retrieving the partial atomic charges (Mullikan population analysis) and constructing the ESP on a uniform grid as $\operatorname{ESP}(\boldsymbol{r})=\Sigma_{\mathrm{i}} q_{\mathrm{i}} /\left|\boldsymbol{r}-\boldsymbol{r}_{\mathrm{i}}\right|$ in atomic units, where $i$ enumerates over atoms. Note that this approximation does not result in any loss in qualitative accuracy compared to the true integral over the electron density (Figure S1).

\section{a. Geometric Properties}
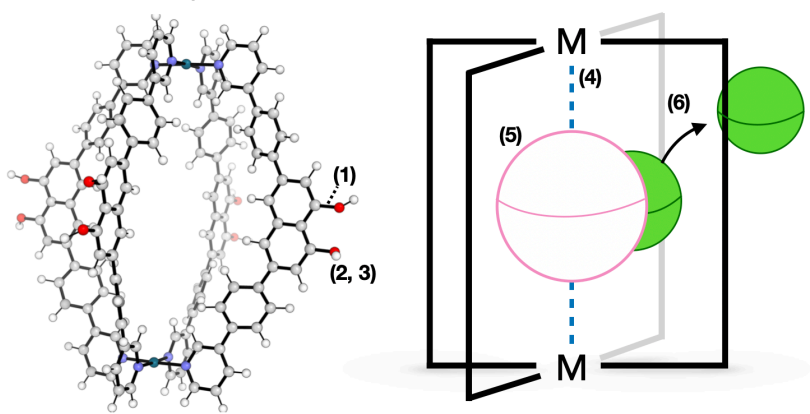

(1) Rotatable bonds $=24$

(4) $\mathrm{M}-\mathrm{M}$ distance $=17.7 \AA$

(2) $\mathrm{H}$-bond donors $=8$

(5) Max enclosed sphere $=213.3 \AA^{3}$

(3) $\mathrm{H}$-bond acceptors $=8$

(6) Max escape sphere $=68.3 \AA^{3}$

\section{b. Electrostatic Potential (ESP)}
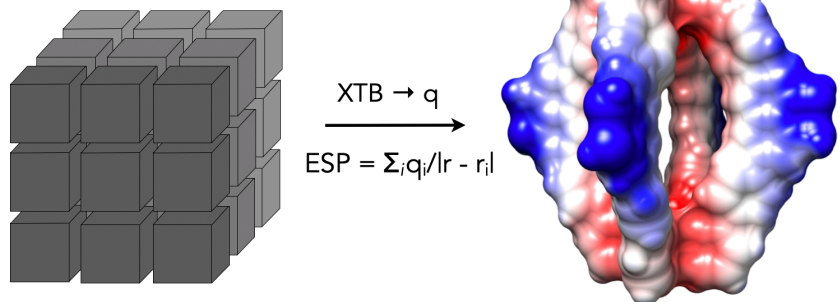

Figure 4. Calculated metallocage properties for an archetype $\mathrm{M}_{2} \mathrm{~L}_{4}$ cage. (a) Geometrical properties: (1) rotatable bonds, (2) H-bond donors, (3) metal-metal distance, (4) maximum enclosed sphere and (5) maximum escape sphere. (b) Electrostatic potential (ESP) calculated from XTB partial atomic charges enumerated over a grid where blue/red corresponds to low/high values of the ESP.

\subsubsection{Substrate Encapsulation and Binding Affinity Prediction}

Once a metallocage is constructed, a substrate can be added to generate a cage-substrate complex. This is achieved by adding the substrate such that its center of mass (COM) is located at the centroid of the cage in a similar way to ridged protein docking. The system is then energy minimized with respect to the substrate rotation 
and conformer, i.e. the global minimum on the rigid-body PES for each conformer is located (Figure 5). To obtain the global minimum a simple (random + BFGS) optimization algorithm is employed, ${ }^{39}$ requiring $\sim n \times m \times O$ energy evaluations, where $n$ is the number of conformers, $m$ the number of initial rotations and $o$ the number of BFGS optimization steps $(\sim 20)$. We have found that $m=50$ affords the global minimum with probability $\sim 0.8$ for the systems tested (Figure S2). The number of conformers to screen is of course substrate dependent and is, as such, user specified.

Considering the large number of energy evaluations that would be required to evaluate binding of each conformer, electronic structure theory is not viable. To speed up this process we instead resort to empirical forcefields (FF). While a number of general FFs are available, their use is labor-intensive, requiring the tabulation of parameters and/or the use of specific molecular simulation packages. Furthermore, inherent approximations in their development limit their accuracy. To circumvent and simplify this problem, and assuming that substratescage interactions are dominated by sterics, we employed the following empirical energy function:

$$
E_{\text {int }}(r+k)=\Sigma_{\mathrm{i}, \mathrm{j}} \in \text { pairs } c \exp \left(a-\left|\boldsymbol{r}_{\mathrm{i}}-\boldsymbol{r}_{\mathrm{j}}\right| / b\right)+k \times S_{\text {atoms }}
$$

Where the first half is a pairwise repulsive term, similar to the one found in a Buckingham potential, with $c=1$ and with units of energy. Using noble gas dimers as as a model for closed shell repulsion, we found that $a$ and $b$ are roughly linearly dependent on the sum of van der Waals radii (Figure S3). The second term is a constant attractive contribution $(k)$ based on the number of substrate atoms $\left(S_{\text {atoms }}\right) \cdot k=0.4 \mathrm{kcal} \mathrm{mol}^{-1}$ atom $^{-1}$ was derived as the optimum classifier for the set of binary binding data outlined in Table S2 (Figure S4). This approximation is considerable, as the attractive terms are dependent on the nature of non-covalent interactions (NCI) between the substrate and the cage (dispersion, H-bonding, etc.). Therefore, we only suggest using this FF to generate cage-substrate complexes where there the attractive term is expected to be small. We refer to this method as 'repulsive' $(r+k)$ and the binding energies arising from it as $E_{\text {int }}(r+k)$ (energy_method='repulsion').

We have also considered a more physical FF, which utilizes repulsive and electrostatic terms. The latter is calculated using either Gasteiger atomic partial charges from RDKit, which do not account for polarization of the atomic centers due to the surrounding environment ( $f e$, energy_method='electrostatic_fast'), or alternatively, charges derived from an XTB single point calculation which account for polarization effects $(e$, energy_method='electrostatic'). These methods more accurately account for NCI between the substrate and the cage. For example, within $\left[\mathrm{Pd}_{2}{ }_{2} \mathrm{~L}_{4}\right]^{4+}$ metallocages, both schemes deliver the expected binding modes, which involves hydrogen bond interactions between the quinone oxygen and the $\alpha-\mathrm{C}-\mathrm{H}$ groups at the top/bottom of the cage (Figure 5). 


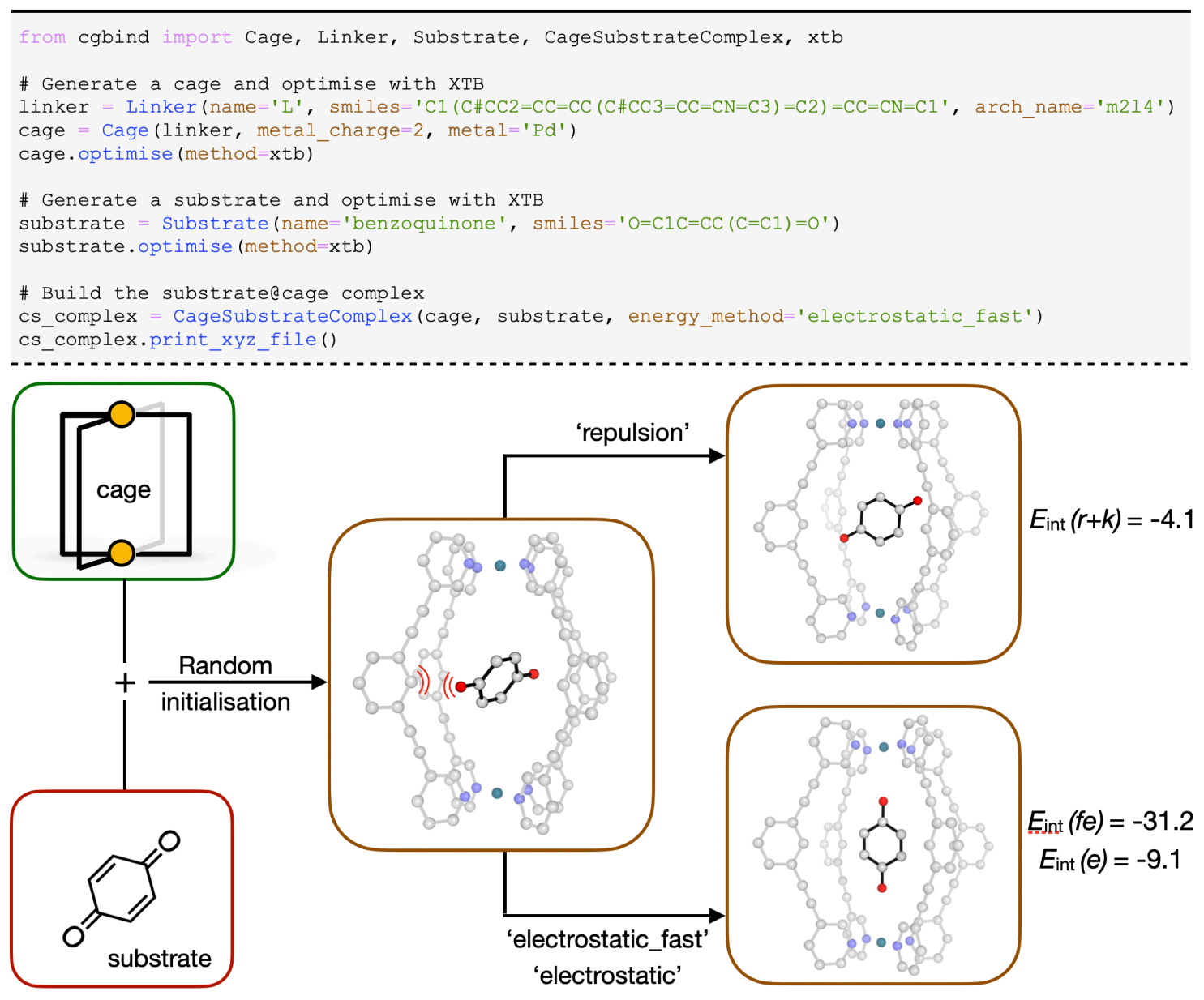

Figure 5. Metallocage-substrate complex construction and interaction energy calculation for benzoquinone inside a $\left[\mathrm{Pd}_{2}{ }_{2} \mathrm{~L}_{4}\right]^{4+}$ cage using the simple repulsive $(r+k)$ and electrostatic forcefields ( $f e$ and $e$ ). Interaction energy predictions are quoted in $\mathrm{kcal} \mathrm{mol}^{-1}$ and do not include solvent, thermal or entropic contributions. Hydrogen atoms omitted for clarity.

Quantifying the binding affinity of a given cage for a substrate is key in determining their applicability in realworld applications. In cgbind, once the cage-substrate complex has been generated, an approximate gas-phase interaction energy $\left(E_{\text {int }}\right)$ based on a simple $\mathrm{FF}$ above is available immediately, as it is minimized in the construction. This gives a rough estimate of the binding affinity and the likelihood of a cage to bind/not-bind a given substrates. A more accurate estimate can be obtained from electronic structure theory calculations, calculating either potential or Gibbs free energies in implicit solvent. The former we recently showed is sufficient to obtain quantitative agreement with experimental binding free energies for $\left[\mathrm{Pd}_{2} \mathrm{~L}_{4}\right]^{4+}$ metallocages. ${ }^{26}$ Key sources of error in the calculation of free energies include inadequate quantification of entropy, sampling and errors in the methodology used. ${ }^{55}$ Solvent effects can be accounted for implicitly using implicit solvent models implemented in electronic structure theory packages and are essential in modulating interaction energies, particularly in polar solvents. Moreover, cgbind provides a starting point for free energy calculations 
required to treat explicitly solvated cavities. The free energy for cavity desolvation and subsequent substrate binding can then be obtained using all-atom molecular dynamics (MD) methods or by adding explicit solvent molecules to the cavity and using the ideal gas model (available in most electronic structure codes).

\subsubsection{Web app GUI}

Alongside the Python interface we have also developed a web application as a GUI to the module. This approach allows a user to interact with key components of the code within a web browser, thereby reducing the activation barrier to its utilization. Furthermore, by developing a web app the user does not need to download or install any packages/dependencies, which in many cases can be limited to specific platforms (Windows/Mac/Linux). The app is currently available at cgbind.chem.ox.ac.uk and provides the ability to: (a) generate and visualize a selection of metallocages, (b) perform analysis of metallocage size, (c) compute and visualize electrostatic potential maps, and (d) perform substrate binding calculations. SMILES strings of linkers can be pasted from chemical software packages, or generated in the cgbind GUI using the 2D drawing tool Kekule.js. ${ }^{56}$ Interactive molecular visualization of the generated structures is enabled using the open-source 3Dmol.js package, allowing useful actions such as rotating and zooming. ${ }^{57}$ The whole app is distributed in a microservice architecture [frontend (Flask), web-server (Gunicorn/nginx), message-broker (Redis), database (PostgreSQL) and task queue (Celery)] using Docker.

\subsection{Examples}

\subsubsection{Geometric Accuracy}

To explore the ability of cgbind to generate cages with varied topologies and linker functionalities we generated nine metallocages (a-i, Figure 6) and computed the root mean squared displacement (RMSD) of the heavy atoms to known crystal structures. With the exception of $\mathbf{L}_{\mathbf{h}}$ (vide infra) all the metallocages were successfully generated from just the linker SMILES string all with RMSD $<1.5 \AA$. Geometry optimization of the structures at the tight-binding DFT level did, in some instances, improve RMSDs while for others (d, $\mathbf{g}, \mathbf{i})$ a larger deviation was obtained with the structure falling into a different local minimum. Similarly, optimization at the DFT level of theory (PBE-D3BJ/def2-SVP) provides no improvement in RMSD. This observation highlights the importance of accounting for the conformational flexibility of the linker and cage when constructing and analysing metallocages and their properties. We believe that this conformational variability provides an interesting avenue to explore substrate-dependent metallocage structure and function as well as a variety of allosteric effects.

To generate a cage, the algorithm requires a conformer with donor atoms in the correct orientation. While conformational generation algorithms available in RDKit (ETKDG, ETDG) are generally adequate, for $\mathbf{L}_{\mathbf{h}}$ (Figure 6), we found that they do not afford a conformer with the torsion parameters required to generate a 
reasonable $\mathrm{M}_{6} \mathrm{~L}_{8}$ metallocage, even with $10^{4}$ conformers requested. Similarly, the open-source conformer generation implemented in OpenBabel (CONFAB), ${ }^{58}$ which performs well at generating structures close to that found in the crystal structure, does not afford the correct conformer from the RDKit initialized 3D structure. ${ }^{59}$ We note that the required conformer of $\mathbf{L}_{\mathbf{h}}$ is $0.6 \mathrm{kcal} \mathrm{mol}^{-1}$ more stable than the first RDKit generated analogue (Figure S5) and therefore should be generated in the conformer ensemble. This highlights the limitation of current conformer-generation algorithms. Therefore, Cage $\mathbf{h}$ was constructed from linker $\mathbf{L}_{\mathbf{h}}$ which was built manually in the correct conformation and a Linker object initialized from an xyz file. Nevertheless, we consider our methodology to be robust in generating metallocages for varying architectures and multiple possible donor atoms. We also note that cgbind provides reasonable structures in seconds rather than in hours (XTB) or weeks (DFT) for the largest metallocages shown in Figure 6.
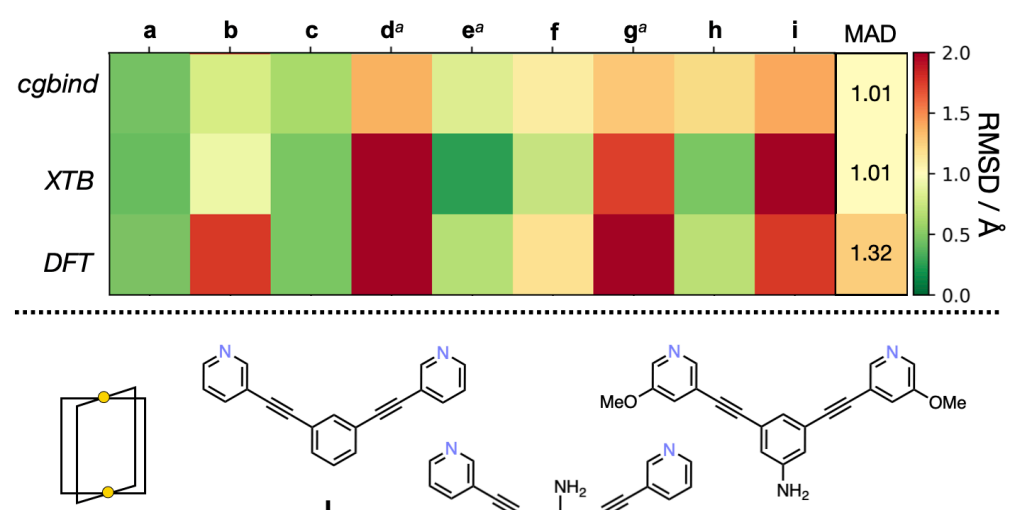

$M_{2} L_{4}$

$\mathrm{L}_{\mathrm{a}}$

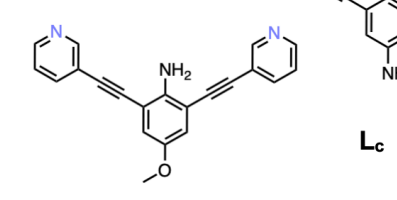

Lb

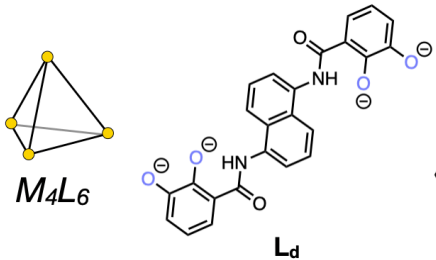

$L_{d}$
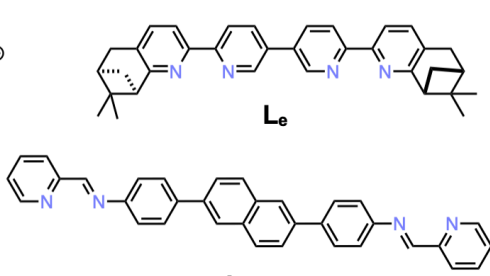

$\mathbf{L}_{\mathrm{f}}$

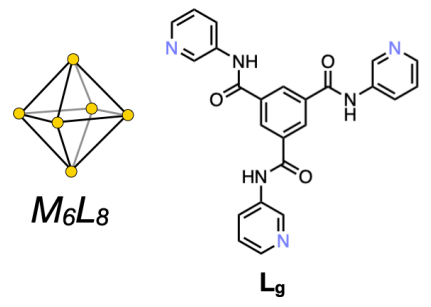

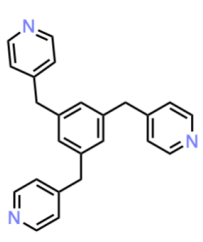

$L_{h}$

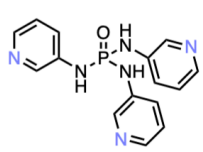

$\mathrm{L}_{\mathrm{i}}$

Figure 6. Root mean squared deviation (RMSD, $\AA$ ) and their mean absolute deviations (MAD) of cgbind-generated metallocage structures relative to available crystal structures in the CSD as a function of increasing computational expense, only heavy atoms were considered for the RMSD calculation. Counterions/solvent molecules in the crystal 
structures were removed for the analysis. Data in Table S1. $a$. DFT geometries converged to a criteria only on forces $\left(\mathrm{RMS}(\mathrm{grad})<5 \mathrm{mHa} \mathrm{a} 0^{-1}\right)$.

\subsubsection{Binding Accuracy}

For 102 host-guest complexes for which either quantitative or qualitative binding information exist in literature we explored the ability of cgbind to generate reasonable host-guest complexes and predict their binding ability. Those systems include $\mathrm{M}_{2} \mathrm{~L}_{4}$ and $\mathrm{M}_{4} \mathrm{~L}_{6}$ host architectures, and a range of polar and apolar substrates and solvents (dichloromethane, acetonitrile and water; Figure 6, Table S2). Specifically, the dataset includes:

1. 12 quinone-type guests and two different $\mathrm{Pd}^{\mathrm{II}}{ }_{2} \mathrm{~L}_{4}$ cages (24 host-guest complexes in total). Their host-guest properties in dichloromethane and acetonitrile have been studied by Lusby et al ${ }^{60}$ Depending on the nature of the quinone guest, binding constants of up to $10^{8} \mathrm{M}^{-1}$ have been observed for these systems. Many of them have also been found to be useful substrates in Diels-Alder reactions and cofactors in radical-cation cycloadditions. ${ }^{48,61}$

2. Six different guests, including polycyclic aromatic hydrocarbons and steroids, with a series of nine aromatic-panelled $\mathrm{Fe}^{\mathrm{II}}{ }_{4} \mathrm{~L}_{6}$ tetrahedral cages in acetonitrile studied by Nitschke et al. (54 hots-guest complexes in total). ${ }^{62}$ In this cage series, the size and arrangement of the aromatic panels were suggested to dictate guest binding propensity.

3. 24 neutral and charged guests bound to $\mathrm{Fe}^{\mathrm{II}}{ }_{4} \mathrm{~L}_{6}$ tetrahedral cages studied by Nitschke et al., ${ }^{63}$ of which 21 were experimentally found to bind with binding constants ranging from 3 up to $10^{4} \mathrm{M}^{-1}$. The host-guest properties of these systems in aqueous solution were found to strongly dependent on the hydrophobicity of the guest.

Cage substrate complexes were initialized using a single conformer in most cases, with the exception of cholesterol where 50 conformers were considered. In all cases reasonable host-guest complexes were obtained. To qualitatively explore binding affinity, and as a first approximation, we consider the experimental data and our prediction as binary classifiers, i.e. either the guest is observed to bind or not within the cage. The binding affinity is considered to be accurate if $E_{\text {int }}<0$ for substrates experimentally reported binds. This approximation assumes that these experimental data can be directly compared and that only host-binding interactions determine binding, omitting the effect of solubility of the substrates and building blocks and the effect of entropy upon binding.

Interestingly, the interaction energies calculated using our simple repulsive $(r+k)$ and both fast and normal electrostatic forcefields ( $f e$ and $e$, respectively) are comparable to those achieved at the tight binding DFT level of theory (XTB) with implicit solvent, and considerably better than single point calculations at the semiempirical PM7-COSMO level of theory, which provides a statistically different prediction and is considerably 
worse than our forcefields and XTB (Figure 7). Optimizations at the PM7 level were not performed as we have previously found that this leads to unphysical metallocage geometries. ${ }^{26}$ Overall our accuracy is modest, $\sim 60 \%$. This includes excellent ( $>80 \%$ ) accuracy for $\mathrm{M}_{2} \mathrm{~L}_{4}$ cages with quinone guests, while for $\mathrm{M}_{4} \mathrm{~L}_{6}$ cages with aromatic guests the accuracy is limited to $\sim 40 \%$ (Figure S8). We hypothesised that the low accuracy of our FF and single point energy evaluations in the $\mathrm{M}_{4} \mathrm{~L}_{6}$ instance was due to the lack of cage relaxation not captured by our ridged body docking approach. To test this, XTB geometry optimisation were performed for the cage, substrate and cage-substrate complexes and the binding affinities evaluated again. In this case, all guests are predicted to bind, leaving the obtained accuracy static at $40 \%$ (Table S4). For a representative example that does not bind experimentally we performed optimisations at the DFT (SMD(MeCN)-M06-2X/def2TZVP//PBE-D3BJ/def2-SVP) level and found that the interaction energy was still negative $\left(E_{\text {int }}=-5 \mathrm{kcal} \mathrm{mol}^{-}\right.$ ${ }^{1}$ ) suggesting that XTB it is not the origin of the limited accuracy (Figure S9). Instead, thermal and entropic contributions, not accounted for here, are expected to shift the binding preference for this series. However, their consideration is known to be costly and subject to major errors. Moreover, the assumption that both host/guest are soluble and therefore equally available in solution should be considered. Nevertheless, our FFs provide accuracy on par with optimisation at the tight binding DFT level in a matter of seconds, with a confidence estimate that suggests true binding substrates are unlikely to be missed (Figure 7b). 

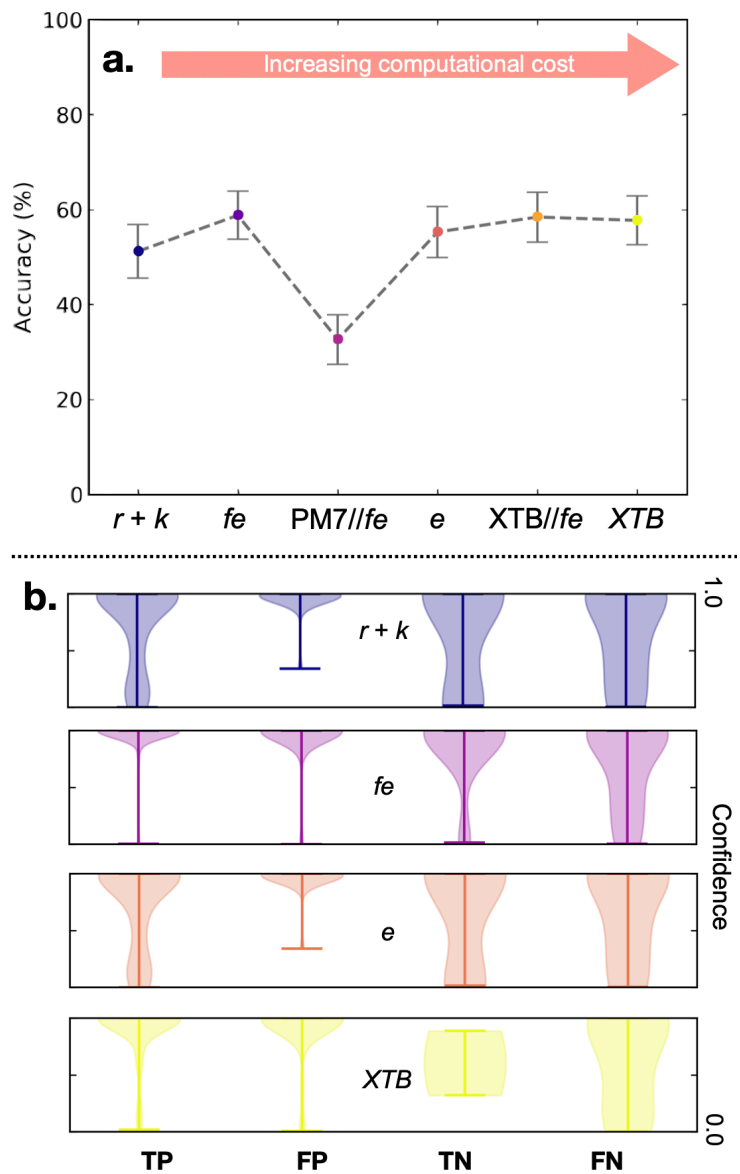

Figure 7. (a) Accuracy (\%) in predicting a substrate binds or not considering 102 host-guest complexes reported in literature. Experimental data tabulated in Table S2. All binding calculations were performed within the cgbind module interfaced through autodE to MOPAC and GFN-XTB. Error bars are plotted as a combined standard deviation, considering a small statistical error $\left(\sim 2 \mathrm{kcal} \mathrm{mol}^{-1}\right.$, Figure S6) with an assumed implicit normal error $\left(\sigma=2 \mathrm{kcal} \mathrm{mol}^{-}\right.$ $\left.{ }^{1}\right)$ and bootstrapping with replacement (10,000 resamples) on the dataset. (b) Confidence for true positives (TP), false positives (FP), true negatives (TN) and false negatives (FN) calculated as: $\mathrm{C}=1-\exp \left(-(\Delta E / k)^{2}\right)$ where $k=5 \mathrm{kcal}$ $\mathrm{mol}^{-1}$.

\subsubsection{Combinatorial Metallocage Library}

To demonstrate the ability of cgbind to construct a library of cages from which subsequent property, screening and filtering calculations can be performed a library of $\mathrm{Pd}_{2} \mathrm{~L}_{4}$ metallocages were generated (available for download as part of the Supporting Information). Employing linkers comprised of three ends (E), 12 links (Z) and 29 centres (C) building blocks, join linearly in a E-Z-C-Z'-E' pattern and $\mathrm{Pd}^{\mathrm{II}}$ as the metal centre, a theoretical total of 13,104 homoleptic cages are possible (building blocks tabulated in Table S3). From these, using linkers initialized with 200 conformers, 5,639 metallocages were constructed with 'reasonable' geometries (defined to be the minimum pairwise distance above $0.8 \AA$, a higher proportion of cages would be constructed in a reasonable geometry were the linkers initialized with more conformers). Considering binding of 
benzoquinone as a guest, cages with cavity radii smaller than the centroid-O distance were removed to afford 1,353. This dataset was further reduced by eliminating those cages that were found to be too large to allow the formation of attractive NCIs between the substrate and the cage (cavity volume $>2 \times$ substrate), leading to 914 cages. Employing the algorithm electrostactic_fast to determine the optimal binding mode, 489 cages were removed based on $E_{\text {int }}>-15 \mathrm{kcal} \mathrm{mol}^{-1}$. Cages with formation energy $\left[\Delta E_{\mathrm{f}}=E_{\mathrm{C}}-\left(2 E_{\mathrm{Pd}}+4 E_{\mathrm{L}}\right)\right]$ above $50 \mathrm{kcal}$ $\mathrm{mol}^{-1}$ of an optimised reference $\mathrm{Pd}_{2} \mathrm{~L}_{4}$ cage reported in ref. 60 calculated using XTB single point calculations were removed affording 275 cages. The most strongly binding hosts $\left(\Delta E_{\text {int }}<-12 \mathrm{kcal} \mathrm{mol}^{-1}\right)$ were then taken forward. Finally, the three most synthetically accessible cages were selected, assuming the most accessible linker molecules lead to the most accessible metallocages, this was determined using the method from ref. 64 (Figure 8). From these core structures monovalent atom functionalization e.g. $\mathrm{H} \rightarrow \mathrm{Me}, \mathrm{Et}, \mathrm{Ph}, \mathrm{CF}_{3}$ etc. may be performed using our Python module for molecular functionalization (github.com/duartegroup/molfunc) to afford a vast library of heteroleptic cages.
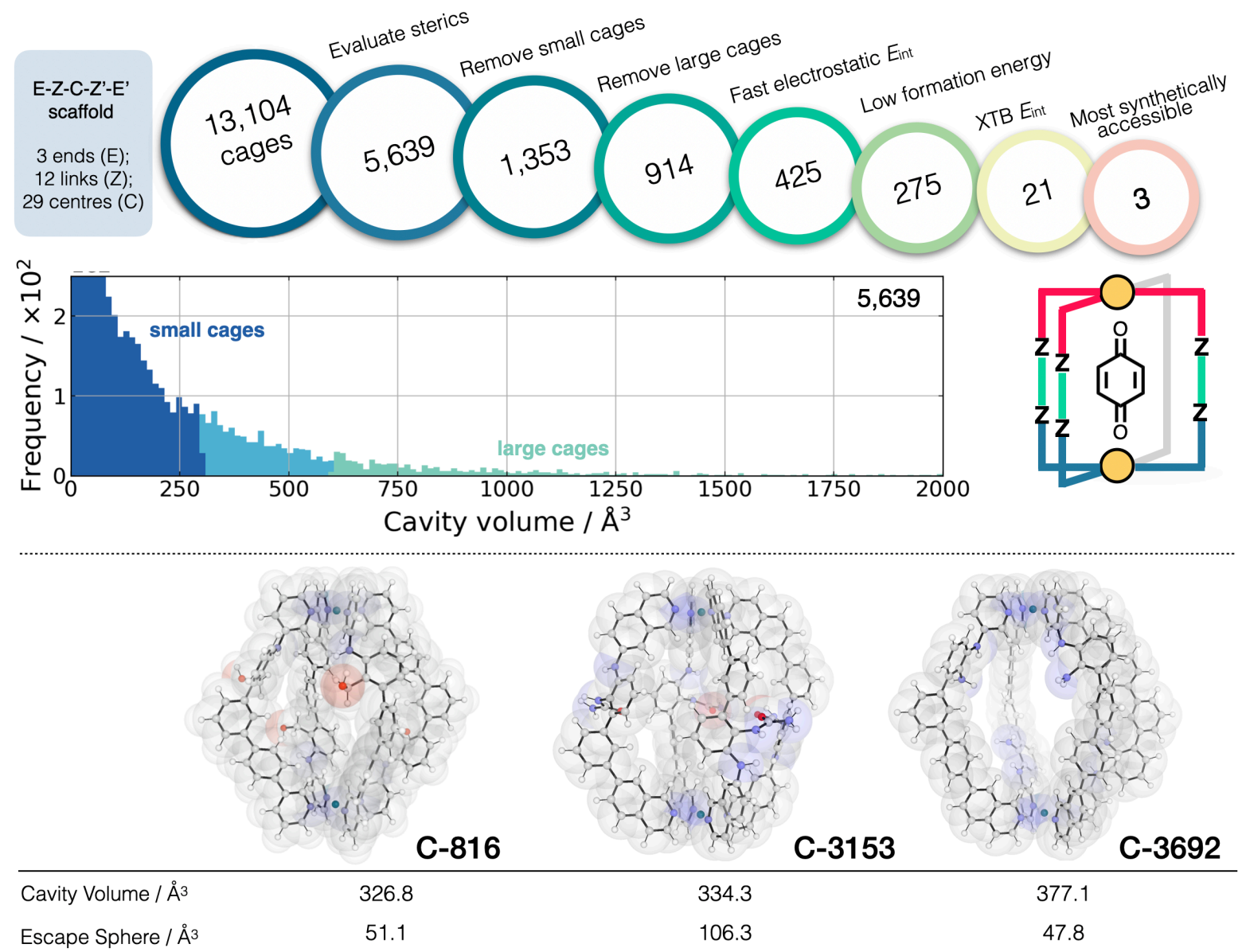

Figure 8. Filtering process used to find the three optimal hosts for benzoquinone based on simple geometric criteria, tight-binding DFT calculations and synthetic accessibility of the constituent linker molecules. Building blocks shown in Figure $\mathbf{S 7 .}$ 


\section{Conclusion}

Here we have described a modular and extensible Python module cgbind for the automated construction and analysis of metallocage structure with arbitrary architecture $\left(\mathrm{M}_{x} \mathrm{~L}_{y}\right)$. Metallocages are constructed using a templating strategy and host-guest complexes generated by minimizing the energy of a simple forcefield developed here. Methods are available to analyze the resulting structures both qualitatively (electrostatic potential) and quantitively (e.g. cavity size, window size) to analyze the resulting structures. We anticipate that cgbind and its web-app (cgbind.chem.ox.ac.uk) will facilitate the rational design of functional metallocage with applications in binding and catalysis. Further work towards the reverse design of metallocages is ongoing, where rather than modifying linkers to subsequently build metallocages and search for tight complexation, the cage is built around the substrate to maximize binding.

\section{Computational Methods.}

Root mean squared deviations (RMSD) were calculated using the Kabsch algorithm implemented in the rmsd Python module, ${ }^{65}$ reordering atoms and not considering hydrogens, all other parameters were set to their defaults. Metallocages had to be approximately aligned manually by fitting metal atoms prior to RMSD calculation, i.e. the reordering of atoms was not completely effective. All cgbind computations were performed with v. 1.0.0 beta. Tight binding (TB-)DFT calculations were performed using GFN2-XTB v. $6.2,{ }^{44}$ PM7 using MOPAC2016, ${ }^{45}$ and resolution of identify DFT using ORCA v. $4.2 \cdot{ }^{46}$ Unless otherwise stated the latter using the PBE functional, ${ }^{66}$ in combination with the D3BJ dispersion correction, ${ }^{67,68}{ }^{\text {def2-SVP basis set }}{ }^{69}$ (which employs effective core potentials for all metals used here $)^{70}$ and the default auxiliary basis sets. ${ }^{71}$

Supporting Information. Information on the empirical force fields, experimental data and estimated interaction energies for the complexes outlined in Figure 7. RMSD values reported in Figure 6 (PDF). Coordinates of geometries obtained from cgbind for the $\left[\mathrm{Pd}_{2} \mathrm{~L}_{4}\right]^{4+}$ benzoquinone complex using the simple repulsive $(r+k)$ and electrostatic forcefields ( $f e$ and $e$ ) (Figure 5) (ZIP). The full library of 5,639 cages is also available at Zenodo: 10.5281/zenodo.3822413. Additional documentation is available at https://duartegroup.github.io/cgbind/.

Corresponding Author: FD: fernanda.duartegonzalez@chem.ox.ac.uk

Author Contributions: TY and RG wrote the code. TY and FD performed the calculations and wrote the manuscript. 
Acknowledgments: We acknowledge the EPSRC Centre for Doctoral Theory and Modelling in Chemical Sciences (EP/L015722/1) for a studentship to TAY generously supported by AWE and for access to the Dirac cluster at Oxford. F.D. thank the Carnegie Trust (RIG007448) and the John Fell Oxford University Press Research Fund (0006752) for financial support. We thank Alistair Sterling and Vicente MartíCentelles for helpful discussions. This work used the Cirrus UK National Tier-2 HPC Service at EPCC (http://www.cirrus.ac.uk) funded by the University of Edinburgh and EPSRC (EP/P020267/1). 


\section{References}

(1) García-Simón, C.; Costas, M.; Ribas, X. Metallosupramolecular Receptors for Fullerene Binding and Release. Chem. Soc. Rev. 2016, 45 (1), 40-62.

(2) Chen, L.; Chen, Q.; Wu, M.; Jiang, F.; Hong, M. Controllable Coordination-Driven Self-Assembly: From Discrete Metallocages to Infinite Cage-Based Frameworks. Acc. Chem. Res. 2015, 48 (2), 201-210.

(3) Pöthig, A.; Casini, A. Recent Developments of Supramolecular Metal-Based Structures for Applications in Cancer Therapy and Imaging. Theranostics 2019, 9 (11), 3150-3169.

(4) Burke, B. P.; Grantham, W.; Burke, M. J.; Nichol, G. S.; Roberts, D.; Renard, I.; Hargreaves, R.; Cawthorne, C.; Archibald, S. J.; Lusby, P. J. Visualizing Kinetically Robust Co III 4 L 6 Assemblies in Vivo : SPECT Imaging of the Encapsulated [ 99m Tc]TcO 4 - Anion. J. Am. Chem. Soc. 2018, 140 (49), $16877-16881$.

(5) Therrien, B.; Süss-Fink, G.; Govindaswamy, P.; Renfrew, A. K.; Dyson, P. J. The "Complex-in-a-Complex" Cations $\left[(\mathrm{Acac})_{2} \mathrm{M} \subset \mathrm{Ru}_{6}\left(\mathrm{p}-\mathrm{IPrC}_{6} \mathrm{H}_{4} \mathrm{Me}\right)_{6}(\mathrm{Tpt})_{2}(\mathrm{Dhbq})_{3}\right]^{6+}$ : A Trojan Horse for Cancer Cells. Angew. Chemie 2008, 120 (20), 3833-3836.

(6) Zava, O.; Mattsson, J.; Therrien, B.; Dyson, P. J. Evidence for Drug Release from a Metalla-Cage Delivery Vector Following Cellular Internalisation. Chem. - A Eur. J. 2010, 16 (5), 1428-1431.

(7) Lewis, J. E. M.; Gavey, E. L.; Cameron, S. A.; Crowley, J. D. Stimuli-Responsive Pd 2 L 4 Metallosupramolecular Cages: Towards Targeted Cisplatin Drug Delivery. Chem. Sci. 2012, 3 (3), 778-784.

(8) Samanta, S. K.; Isaacs, L. Biomedical Applications of Metal Organic Polygons and Polyhedra (MOPs). Coord. Chem. Rev. 2020, 410, 213181.

(9) Sepehrpour, H.; Fu, W.; Sun, Y.; Stang, P. J. Biomedically Relevant Self-Assembled Metallacycles and Metallacages. J. Am. Chem. Soc. 2019, 141 (36), 14005-14020.

(10) Casini, A.; Woods, B.; Wenzel, M. The Promise of Self-Assembled 3D Supramolecular Coordination Complexes for Biomedical Applications. Inorg. Chem. 2017, 56 (24), 14715-14729.

(11) Fang, Y.; Powell, J. A.; Li, E.; Wang, Q.; Perry, Z.; Kirchon, A.; Yang, X.; Xiao, Z.; Zhu, C.; Zhang, L.; Huang, F.; Zhou, H.-C. Catalytic Reactions within the Cavity of Coordination Cages. Chem. Soc. Rev. 2019, 48 (17), 4707-4730.

(12) Desmarets, C.; Ducarre, T.; Rager, M.; Gontard, G.; Amouri, H. Self-Assembled M2L4 Nanocapsules: Synthesis, Structure and Host-Guest Recognition Toward Square Planar Metal Complexes. Materials (Basel). 2014, 7 (1), 287-301.

(13) Chakrabarty, R.; Mukherjee, P. S.; Stang, P. J. Supramolecular Coordination: Self-Assembly of Finite Twoand Three-Dimensional Ensembles. Chem. Rev. 2011, 111 (11), 6810-6918.

(14) Segura, J. L.; Royuela, S.; Mar Ramos, M. Post-Synthetic Modification of Covalent Organic Frameworks. Chem. Soc. Rev. 2019, 48 (14), 3903-3945.

(15) Erythropel, H. C.; Zimmerman, J. B.; de Winter, T. M.; Petitjean, L.; Melnikov, F.; Lam, C. H.; Lounsbury, A. W.; Mellor, K. E.; Janković, N. Z.; Tu, Q.; Pincus, L. N.; Falinski, M. M.; Shi, W.; Coish, P.; Plata, D. L.; Anastas, P. T. The Green ChemisTREE: 20 Years after Taking Root with the 12 Principles. Green Chem. 
2018, 20 (9), 1929-1961.

(16) Poree, C.; Schoenebeck, F. A Holy Grail in Chemistry: Computational Catalyst Design: Feasible or Fiction? Acc. Chem. Res. 2017, 50 (3), 605-608.

(17) Neese, F.; Atanasov, M.; Bistoni, G.; Maganas, D.; Ye, S. Chemistry and Quantum Mechanics in 2019: Give Us Insight and Numbers. J. Am. Chem. Soc. 2019, 141 (7), 2814-2824.

(18) Xu, J.; Hagler, A. Chemoinformatics and Drug Discovery. Molecules 2002, 7 (8), 566-600.

(19) Durand, D. J.; Fey, N. Computational Ligand Descriptors for Catalyst Design. Chem. Rev. 2019, 119 (11), 6561-6594.

(20) Greenaway, R. L.; Santolini, V.; Bennison, M. J.; Alston, B. M.; Pugh, C. J.; Little, M. A.; Miklitz, M.; Eden-Rump, E. G. B.; Clowes, R.; Shakil, A.; Cuthbertson, H. J.; Armstrong, H.; Briggs, M. E.; Jelfs, K. E.; Cooper, A. I. High-Throughput Discovery of Organic Cages and Catenanes Using Computational Screening Fused with Robotic Synthesis. Nat. Commun. 2018, 9 (1), 2849.

(21) Freeze, J. G.; Kelly, H. R.; Batista, V. S. Search for Catalysts by Inverse Design: Artificial Intelligence, Mountain Climbers, and Alchemists. Chem. Rev. 2019, 119 (11), 6595-6612.

(22) Foscato, M.; Jensen, V. R. Automated in Silico Design of Homogeneous Catalysts. ACS Catal. 2020, 10 (3), $2354-2377$.

(23) Rosales, A. R.; Wahlers, J.; Limé, E.; Meadows, R. E.; Leslie, K. W.; Savin, R.; Bell, F.; Hansen, E.; Helquist, P.; Munday, R. H.; Wiest, O.; Norrby, P.-O. Rapid Virtual Screening of Enantioselective Catalysts Using CatVS. Nat. Catal. 2019, 2 (1), 41-45.

(24) Guan, Y.; Ingman, V. M.; Rooks, B. J.; Wheeler, S. E. AARON: An Automated Reaction Optimizer for New Catalysts. J. Chem. Theory Comput. 2018, 14 (10), 5249-5261.

(25) Hachmann, J.; Olivares-Amaya, R.; Atahan-Evrenk, S.; Amador-Bedolla, C.; Sánchez-Carrera, R. S.; GoldParker, A.; Vogt, L.; Brockway, A. M.; Aspuru-Guzik, A. The Harvard Clean Energy Project: Large-Scale Computational Screening and Design of Organic Photovoltaics on the World Community Grid. J. Phys. Chem. Lett. 2011, 2 (17), 2241-2251.

(26) Young, T. A.; Martí-Centelles, V.; Wang, J.; Lusby, P. J.; Duarte, F. Rationalizing the Activity of an “Artificial Diels-Alderase": Establishing Efficient and Accurate Protocols for Calculating Supramolecular Catalysis. J. Am. Chem. Soc. 2020, 142 (3), 1300-1310.

(27) Wilmer, C. E.; Leaf, M.; Lee, C. Y.; Farha, O. K.; Hauser, B. G.; Hupp, J. T.; Snurr, R. Q. Large-Scale Screening of Hypothetical Metal-Organic Frameworks. Nat. Chem. 2012, 4 (2), 83-89.

(28) Simon, C. M.; Mercado, R.; Schnell, S. K.; Smit, B.; Haranczyk, M. What Are the Best Materials To Separate a Xenon/Krypton Mixture? Chem. Mater. 2015, 27 (12), 4459-4475.

(29) Ong, S. P.; Richards, W. D.; Jain, A.; Hautier, G.; Kocher, M.; Cholia, S.; Gunter, D.; Chevrier, V. L.; Persson, K. A.; Ceder, G. Python Materials Genomics (Pymatgen): A Robust, Open-Source Python Library for Materials Analysis. Comput. Mater. Sci. 2013, 68, 314-319.

(30) Willems, T. F.; Rycroft, C. H.; Kazi, M.; Meza, J. C.; Haranczyk, M. Algorithms and Tools for HighThroughput Geometry-Based Analysis of Crystalline Porous Materials. Microporous Mesoporous Mater. 
2012, 149 (1), 134-141.

(31) Bucior, B. J.; Rosen, A. S.; Haranczyk, M.; Yao, Z.; Ziebel, M. E.; Farha, O. K.; Hupp, J. T.; Siepmann, J. I.; Aspuru-Guzik, A.; Snurr, R. Q. Identification Schemes for Metal-Organic Frameworks To Enable Rapid Search and Cheminformatics Analysis. Cryst. Growth Des. 2019, 19 (11), 6682-6697.

(32) Rosen, A. S.; Notestein, J. M.; Snurr, R. Q. Identifying Promising Metal-Organic Frameworks for Heterogeneous Catalysis via High-Throughput Periodic Density Functional Theory. J. Comput. Chem. 2019, 40 (12), 1305-1318.

(33) Miklitz, M.; Jiang, S.; Clowes, R.; Briggs, M. E.; Cooper, A. I.; Jelfs, K. E. Computational Screening of Porous Organic Molecules for Xenon/Krypton Separation. J. Phys. Chem. C 2017, 121 (28), 15211-15222.

(34) Turcani, L.; Berardo, E.; Jelfs, K. E. Stk: A Python Toolkit for Supramolecular Assembly. J. Comput. Chem. 2018, 39 (23), 1931-1942.

(35) Sturluson, A.; Huynh, M. T.; York, A. H. P.; Simon, C. M. Eigencages: Learning a Latent Space of Porous Cage Molecules. ACS Cent. Sci. 2018, 4 (12), 1663-1676.

(36) Kravchenko, O.; Varava, A.; Pokorny, F. T.; Devaurs, D.; Kavraki, L. E.; Kragic, D. A Robotics-Inspired Screening Algorithm for Molecular Caging Prediction. J. Chem. Inf. Model. 2020, 60 (3), 1302-1316.

(37) Miklitz, M.; Jelfs, K. E. Pywindow : Automated Structural Analysis of Molecular Pores. J. Chem. Inf. Model. 2018, 58 (12), 2387-2391.

(38) van der Walt, S.; Colbert, S. C.; Varoquaux, G. The NumPy Array: A Structure for Efficient Numerical Computation. Comput. Sci. Eng. 2011, 13 (2), 22-30.

(39) Virtanen, P.; Gommers, R.; Oliphant, T. E.; Haberland, M.; Reddy, T.; Cournapeau, D.; Burovski, E.; Peterson, P.; Weckesser, W.; Bright, J.; van der Walt, S. J.; Brett, M.; Wilson, J.; Millman, K. J.; Mayorov, N.; Nelson, A. R. J.; Jones, E.; Kern, R.; Larson, E.; Carey, C.; Polat, I.; Feng, Y.; Moore, E. W.; VanderPlas, J.; Laxalde, D.; Perktold, J.; Cimrman, R.; Henriksen, I.; Quintero, E. A.; Harris, C. R.; Archibald, A. M.; Ribeiro, A. H.; Pedregosa, F.; van Mulbregt, P.; Contributors, S. 1. 0. SciPy 1.0-Fundamental Algorithms for Scientific Computing in Python. Nature Methods, 2020, 17, (3), 261-272.

(40) Hagberg, A.; D, S.; Swart, P. Exploring Network Structure, Dynamics, and Function Using NetworkX. In Proceedings of the 7th Python in Science conference; 2008; pp 11-15.

(41) Weininger, D. SMILES, a Chemical Language and Information System. 1. Introduction to Methodology and Encoding Rules. J. Chem. Inf. Model. 1988, 28 (1), 31-36.

(42) Landrum, G. RDKit: Open-Source Cheminformatics, v. 2019; GitHub (https://github.com/rdkit/rdkit), 2019.

(43) Riniker, S.; Landrum, G. A. Better Informed Distance Geometry: Using What We Know To Improve Conformation Generation. J. Chem. Inf. Model. 2015, 55 (12), 2562-2574.

(44) Bannwarth, C.; Ehlert, S.; Grimme, S. GFN2-XTB-An Accurate and Broadly Parametrized Self-Consistent Tight-Binding Quantum Chemical Method with Multipole Electrostatics and Density-Dependent Dispersion Contributions. J. Chem. Theory Comput. 2019, 15 (3), 1652-1671.

(45) Stewart, J. J. P. MOPAC2016; Stewart Computational Chemistry: Colorado Springs, CO, USA, 2016.

(46) Neese, F. Software Update: The ORCA Program System, Version 4.0. WIREs Comput. Mol. Sci. 2018, 8 
(1), 1-6.

(47) Valiev, M.; Bylaska, E. J.; Govind, N.; Kowalski, K.; Straatsma, T. P.; Van Dam, H. J. J.; Wang, D.; Nieplocha, J.; Apra, E.; Windus, T. L.; de Jong, W. A. NWChem: A Comprehensive and Scalable OpenSource Solution for Large Scale Molecular Simulations. Comput. Phys. Commun. 2010, 181 (9), $1477-$ 1489.

(48) Martí-Centelles, V.; Lawrence, A. L.; Lusby, P. J. High Activity and Efficient Turnover by a Simple, SelfAssembled “Artificial Diels-Alderase." J. Am. Chem. Soc. 2018, 140 (8), 2862-2868.

(49) Kabsch, W. A Solution for the Best Rotation to Relate Two Sets of Vectors. Acta Crystallogr. Sect. A 1976, $32(5), 922-923$.

(50) Caulder, D. L.; Powers, R. E.; Parac, T. N.; Raymond, K. N. The Self-Assembly of a Predesigned Tetrahedral M4L6 Supramolecular Cluster. Angew. Chemie Int. Ed. 1998, 37 (13-14), 1840-1843.

(51) Zhu, R.; Bloch, W. M.; Holstein, J. J.; Mandal, S.; Schäfer, L. V.; Clever, G. H. Donor-Site-Directed Rational Assembly of Heteroleptic Cis -[Pd 2 L 2 L' 2 ] Coordination Cages from Picolyl Ligands. Chem. A Eur. J. 2018, 24 (49), 12976-12982.

(52) Young, D. C. Computational Chemistry; John Wiley \& Sons, Inc.: New York, USA, 2001.

(53) Wales, D. J.; Doye, J. P. K. Global Optimization by Basin-Hopping and the Lowest Energy Structures of Lennard-Jones Clusters Containing up to 110 Atoms. J. Phys. Chem. A 1997, 101 (28), 5111-5116.

(54) Grimme, S.; Bannwarth, C.; Shushkov, P. A Robust and Accurate Tight-Binding Quantum Chemical Method for Structures, Vibrational Frequencies, and Noncovalent Interactions of Large Molecular Systems Parametrized for All Spd-Block Elements ( $\mathrm{Z}=1-86)$. J. Chem. Theory Comput. 2017, 13 (5), 1989-2009.

(55) Zhou, H.-X.; Gilson, M. K. Theory of Free Energy and Entropy in Noncovalent Binding. Chem. Rev. 2009, 109 (9), 4092-4107.

(56) Jiang, C.; Jin, X.; Dong, Y.; Chen, M. Kekule.Js: An Open Source JavaScript Chemoinformatics Toolkit. J. Chem. Inf. Model. 2016, 56 (6), 1132-1138.

(57) Rego, N.; Koes, D. 3Dmol.Js: Molecular Visualization with WebGL. Bioinformatics 2015, 31 (8), 1322 1324.

(58) O’Boyle, N. M.; Vandermeersch, T.; Flynn, C. J.; Maguire, A. R.; Hutchison, G. R. Confab - Systematic Generation of Diverse Low-Energy Conformers. J. Cheminform. 2011, 3 (1), 8.

(59) Ebejer, J.-P.; Morris, G. M.; Deane, C. M. Freely Available Conformer Generation Methods: How Good Are They? J. Chem. Inf. Model. 2012, 52 (5), 1146-1158.

(60) August, D. P.; Nichol, G. S.; Lusby, P. J. Maximizing Coordination Capsule-Guest Polar Interactions in Apolar Solvents Reveals Significant Binding. Angew. Chemie Int. Ed. 2016, 55 (48), 15022-15026.

(61) Spicer, R. L.; Stergiou, A. D.; Young, T. A.; Duarte, F.; Symes, M. D.; Lusby, P. J. Host-Guest-Induced Electron Transfer Triggers Radical-Cation Catalysis. J. Am. Chem. Soc. 2020, 142 (5), 2134-2139.

(62) Ronson, T. K.; Meng, W.; Nitschke, J. R. Design Principles for the Optimization of Guest Binding in Aromatic-Paneled Fe II 4 L 6 Cages. J. Am. Chem. Soc. 2017, 139 (28), 9698-9707.

(63) Smulders, M. M. J.; Zarra, S.; Nitschke, J. R. Quantitative Understanding of Guest Binding Enables the 
Design of Complex Host-Guest Behavior. J. Am. Chem. Soc. 2013, 135 (18), 7039-7046.

(64) Ertl, P.; Schuffenhauer, A. Estimation of Synthetic Accessibility Score of Drug-like Molecules Based on Molecular Complexity and Fragment Contributions. J. Cheminform. 2009, 1 (1), 8.

(65) Kromann, J. C. Calculate Root-Mean-Square Deviation (RMSD) of Two Molecules Using Rotation. v.1.3.0; GitHub (https://github.com/charnley/rmsd), 2019.

(66) Perdew, J. P.; Burke, K.; Ernzerhof, M. Generalized Gradient Approximation Made Simple. Phys. Rev. Lett. 1996, 77 (18), 3865-3868.

(67) Grimme, S.; Antony, J.; Ehrlich, S.; Krieg, H. A Consistent and Accurate Ab Initio Parametrization of Density Functional Dispersion Correction (DFT-D) for the 94 Elements H-Pu. J. Chem. Phys. 2010, 132 (15), 154104.

(68) Grimme, S.; Ehrlich, S.; Goerigk, L. Effect of the Damping Function in Dispersion Corrected Density Functional Theory. J. Comput. Chem. 2011, 32 (7), 1456-1465.

(69) Weigend, F.; Ahlrichs, R. Balanced Basis Sets of Split Valence, Triple Zeta Valence and Quadruple Zeta Valence Quality for H to Rn: Design and Assessment of Accuracy. Phys. Chem. Chem. Phys. 2005, 7 (18), 3297- 3305.

(70) Andrae, D.; Häußermann, U.; Dolg, M.; Stoll, H.; Preuß, H. Energy-Adjusted Ab Initio Pseudopotentials for the Second and Third Row Transition Elements. Theor. Chim. Acta 1990, 77 (2), 123-141.

(71) Weigend, F. Accurate Coulomb-Fitting Basis Sets for H to Rn. Phys. Chem. Chem. Phys. 2006, 8 (9), 10571065. 
Table of Contents

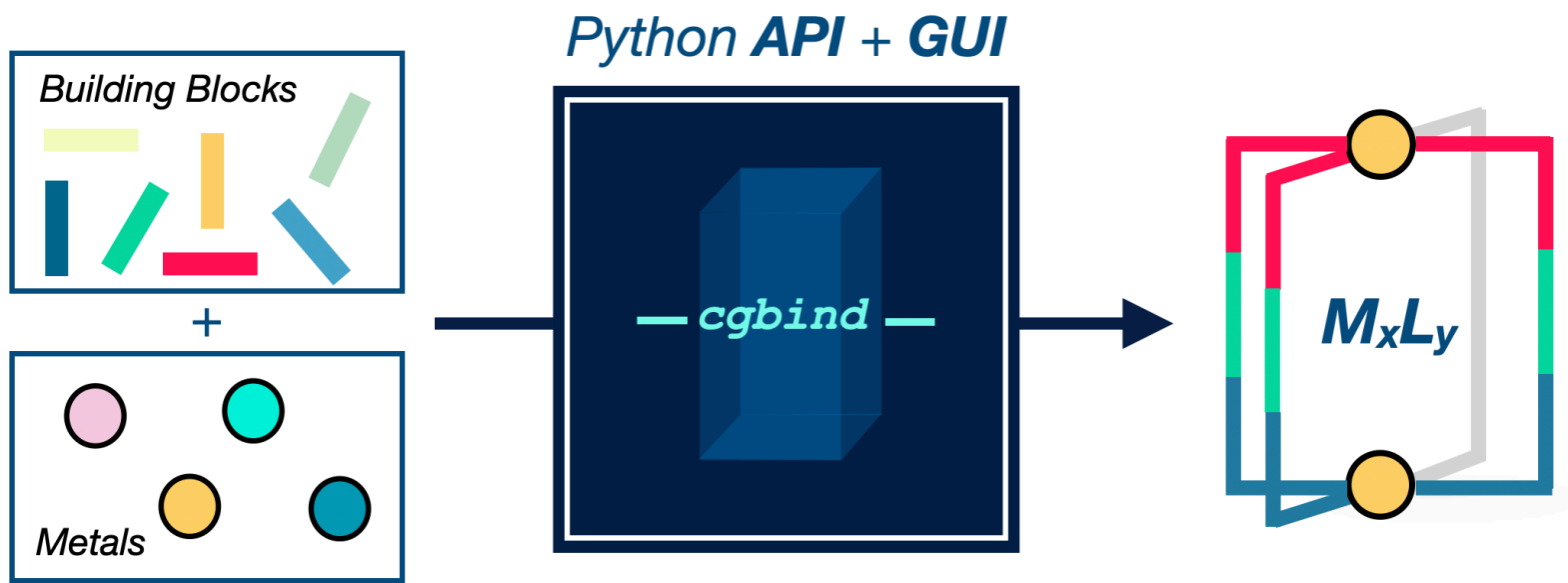

Accurate Geometries • Automated Calculations - Host-Guest Complexes 